\title{
A Cross-Sectional Piecewise Constant Model for Segmenting Highly Curved Fiber Tracts in Diffusion MR Images
}

\author{
Brian G. Booth and Ghassan Hamarneh \\ Medical Image Analysis Lab, Simon Fraser University, Burnaby, Canada \\ bgb2@sfu.ca, hamarneh@sfu.ca
}

\begin{abstract}
We propose a cross-sectional piecewise constant model for the segmentation of highly curved fiber tracts in diffusion MRI scans. An "anchor curve", obtained via tractography, provides the overall shape of the tract and allows us to examine the tract's microstructure at the level of cross-sectional planes normal to the curve. Each cross-section is modeled as a piecewise constant image, allowing us to address changes in measured diffusion due to the curving of the tract while still capturing overall tract structure. Results on both synthetic and real data show improved segmentation quality compared to state-of-the-art methods, particularly in areas of crossing fibers.
\end{abstract}

\section{Introduction}

In diffusion MRI (dMRI), segmentation is often used to delineate axonal fiber tracts connecting functional brain regions [12]. Initial attempts to segment fiber tracts focused around performing streamline tractography, then defining the segment as the set of voxels that contain the streamlines (e.g., [16]). However, the goal of tractography is to capture a tract's direction and orientation, not its width. As a result, tractography cannot capture fine details along the surface of a fiber tract, routinely leading to under-segmentation [3]. Instead of relying on a collection of 3D streamlines with an unclear encapsulating surface, segmentation algorithms that label the underlying 3D image domain are preferred for defining a tract's volumetric region. Among these volumetric dMRI segmentation algorithms, many assume a piecewise-constant model of the image [9, 11]. However, given the fact that dMRI data contains tract orientation information, the success of these piecewise constant approaches is limited to tracts that have little curvature (e.g., corpus callosum [9]).

Segmenting highly curved tracts in dMRI scans requires extending segmentation techniques to handle a tract's variable appearance. This can be done by either increasing the complexity of the image model (e.g., piecewise smooth [17]) or by pre-processing the dMRI scan so that a simpler segmentation model can be applied effectively. The latter approach has been more popular over the past decade with examples including segmentation based on pre-computed edge information [10] and clustering voxels using local statistics pre-computed from Parzen 
windows [2]. More recently, tractography results have been used to provide global tract shape information as input to the segmentation process [3, 13, 15], allowing for the pre-processing of an image based on the orientation of the tract. This global shape information may well complement the local appearance information obtained using Parzen windowing or edge maps, yet, individually, these approaches are limited by either susceptibility to noise or lack of fidelity between the data and the image model [13].

We propose that a hybrid approach, where local appearance information is combined with global shape information, can show increased segmentation accuracy for diffusion MR images. We base this hybrid algorithm on the assumption that a tract's cross-section (i.e., the plane perpendicular to the tract's local direction) shows relatively constant diffusion compared to its surroundings. The piecewise constancy assumption is then justifiably applied only at a local scale while, at the global scale, cross-sectional planes are defined based on an "anchor curve" obtained from tractography. Results on both synthetic and real data show improved segmentation quality compared to state-of-the-art methods [2, 9 11, 15], particularly in areas of crossing fiber tracts.

\section{Methods}

Figure 1 displays the general workflow of our segmentation algorithm. Like in the work of Niethammer et al. [15], we begin by generating an anchor curve from a tractography algorithm. We also end by segmenting a simplified version of the diffusion MR image. Where we differ is in how we generate that simplified image. In [15], Niethammer et al. reorient tensors by the curvature of the anchor

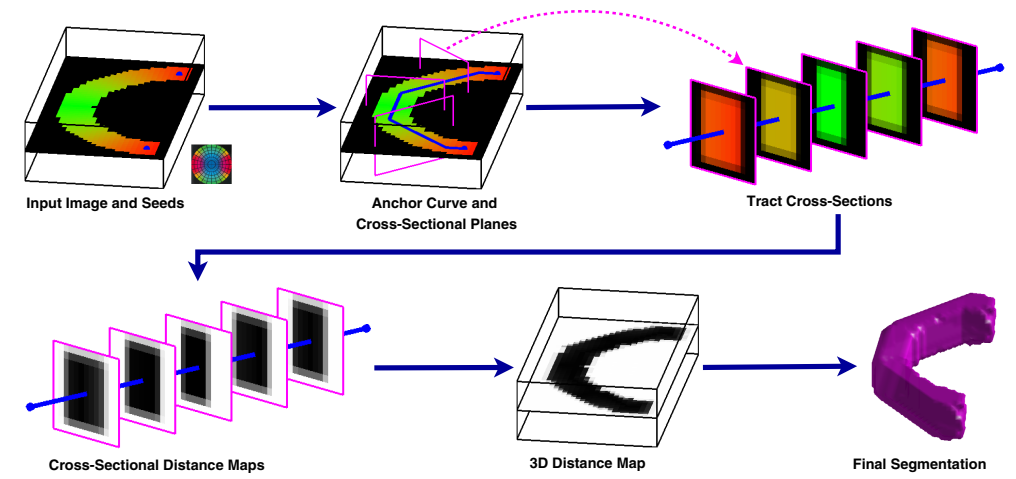

Fig. 1. Our proposed segmentation workflow. Tractography is employed to generate an anchor curve (blue) which is then used to generate cross-sections of the fiber bundle (magenta). For each cross-section, we measure diffusion dissimilarities between the points on the plane and the intersection point between the plane and the anchor curve. These dissimilarities are then interpolated back into a 3D image and a scalar segmentation algorithm provides us with the final segmentation. 
curve, then assume a global piecewise-constant image model. We instead assume a piecewise-constant model only on cross-sectional planes normal to the anchor curve. The following subsections describe how we employ that model to simplify and segment diffusion MR images.

Anchor Curve Generation: Given a dMR image $\mathcal{I}: \Omega \rightarrow \mathcal{M}$ that maps a point $\mathbf{x}$ in our image space $\Omega \subset \mathbb{R}^{3}$ to a diffusion representation (e.g., tensor, ODF) on a manifold $\mathcal{M}$, we can generate an anchor curve $\mathbf{r}:[0,1] \rightarrow \Omega$ from various tractography algorithms. In this work, we employ the minimal path tractography algorithm of Zalesky [18] to generate r. Edge weights for the graph used by Zalesky's algorithm were computed analytically using [6].

Obtaining Tract Cross-Sections: For each point $s$ along the given anchor curve $\mathbf{r}$, we compute the Frenet frame defined by the curve's local tangent $\mathbf{T}$, normal $\mathbf{N}$, and binormal $\mathbf{B}$ vectors

$$
\mathbf{T}=\frac{\partial \mathbf{r}}{\partial s} \quad \mathbf{N}=\frac{\frac{\partial \mathbf{T}}{\partial s}}{\left\|\frac{\partial \mathbf{T}}{\partial s}\right\|} \quad \mathbf{B}=\mathbf{T} \times \mathbf{N} .
$$

The resulting normal and binormal vectors span (and parameterize) the crosssectional plane normal to $\mathbf{r}$. For points $s_{i}$ on the anchor curve $\mathbf{r}$ where $\partial \mathbf{T} / \partial s=0$, we use the closest stable Frenet frame of $\mathbf{r}$ rotated so that is tangent vector aligns with the tangent vector at $s_{i}$.

Given the local Frenet frame $\{\mathbf{T}, \mathbf{N}, \mathbf{B}\}$ and a point $s$ on the anchor curve $\mathbf{r}$, we generate points $\mathbf{x} \in \Omega$ on the cross-sectional plane by sampling along the normal and binormal vectors:

$$
\mathbf{x}=\mathbf{r}(s)+u * \mathbf{N}+v * \mathbf{B} .
$$

The diffusion representation (e.g., tensor, ODF) at $\mathbf{x}$ - and correspondingly $(u, v)$ in the cross-sectional image space $\Phi_{s}-$ is linearly interpolated from the original image $\mathcal{I}$. This procedure produces our cross-sectional images $I_{s}: \Phi_{s} \rightarrow$ $\mathcal{M}$ and the corresponding $3 \mathrm{D}$ coordinates of each cross-sectional image pixel $\Pi_{s}: \Phi_{s} \rightarrow \Omega$.

Cross-Sectional Piecewise Constancy: Our approach is based on the assumption that the diffusion data within a cross-section of a fiber bundle can be well-modeled using a piecewise-constant function. Given that a fiber tract is a collection of coherently aligned axons, we expect the diffusion within the cross-section of the tract to be similar to that at the plane's intersection with the anchor curve. Meanwhile, we expect diffusion on that cross-sectional plane but outside the fiber tract to be different from that at the plane's anchor curve intersection point. As a result, computing dissimilarities between the diffusion data on the anchor curve $\mathcal{I}(\mathbf{r}(s))$ and the diffusion data throughout the crosssection $I_{s}$ will provide us with a scalar feature $D_{s}$ that will correlate well with fiber bundle membership. 
Various dissimilarity metrics can be employed, including those for second order tensors (e.g., [1]), $4^{\text {th }}$-order tensors [5], and spherical harmonic ODF representations [8]. Given a chosen metric $d(\cdot)$, we employ the following mapping

$$
D_{s}(u, v)=\log \left(\frac{d\left(I_{s}(u, v), \mathcal{I}(\mathbf{r}(s))\right)}{F A(\mathcal{I}(\mathbf{r}(s)))}+\epsilon\right)
$$

which applies a log mapping to the dMRI dissimilarities and normalizes them by the fractional anisotropy (FA) of the point intersecting the anchor tract. We found, following empirical examination, that the log-mapping leads to more Gaussian-distributed dissimilarities inside and outside the tract of interest while dividing by the anchor curve's FA helps normalize the range of dissimilarities from one cross-section to another. Note that since the anchor curve was obtained via tractography, its FA will be greater than zero.

Mapping Dissimilarities to the Image Space: Once we have the tensor distance feature $D_{s}$ computed from (3) for a collection of points $\Pi_{s}$ defined by (2), we proceed with reconstructing a 3D distance image. This task is a basic scattered data interpolation problem and we employ an approach based on radial basis functions and k-nearest neighbours. The interpolated 3D dissimilarity map $\mathcal{D}$ is given as

$$
\mathcal{D}(\mathbf{x})=\sum_{i=1}^{k} \frac{\exp \left(-\left\|\Pi_{s(i)}(u(i), v(i))-\mathbf{x}\right\|\right)}{\sum_{j=1}^{k} \exp \left(-\left\|\Pi_{s(j)}(u(j), v(j))-\mathbf{x}\right\|\right)} D_{s(i)}(u(i), v(i))
$$

where $(u(i), v(i))$ in cross section $s(i)$ is the $i^{\text {th }}$ nearest neighbour to $\mathbf{x}$ in $\Omega$.

Dissimilarity Map Segmentation: Using the local diffusion dissimilarities from (3), we have reduced our dMR image, with its variable region appearance and manifold-valued data, to a scalar image that is well modeled by a piecewise constant function. As a result, it now makes sense to employ a piecewise constant segmentation algorithm. We use a probabilistic variant of the Chan-Vese segmentation algorithm that minimizes

$$
\begin{aligned}
E\left(\mathcal{S}, \mu_{\text {in }}, \sigma_{\text {in }}, \mu_{\text {out }}, \sigma_{\text {out }}\right)= & \alpha \int_{\partial \mathcal{S}} d \mathbf{x}+\beta \int_{\mathbf{x} \in \mathcal{S}}-\log \left(p\left(\mathbf{x} \mid \mu_{\text {in }}, \sigma_{\text {in }}\right)\right) d \mathbf{x}+ \\
& \beta \int_{\mathbf{x} \notin \mathcal{S}}-\log \left(p\left(\mathbf{x} \mid \mu_{\text {out }}, \sigma_{\text {out }}\right)\right) d \mathbf{x}
\end{aligned}
$$

where $\mathcal{S} \subset \Omega$ is the segmentation, $\mu_{\text {in }}, \sigma_{\text {in }}\left(\mu_{\text {out }}, \sigma_{\text {out }}\right)$ represent the mean and standard deviation of distances $D$ inside (outside) $\mathcal{S}$, and weights $\alpha, \beta$ regulate the trade-off between the contour regularization and image fidelity terms. We optimize the segmentation energy in (5) using the total variational approach of Bresson et al. [7]. Note that this is the same optimization scheme used by the competing approach of Niethammer et al. [15] though they use it to segment images of diffusion tensor primary eigenvectors. As with the approach of Niethammer et al., all voxels a distance greater than $d_{\max }$ from the anchor curve are set to belong to the background while the foreground segment containing the anchor curve is taken as the final segmentation. 


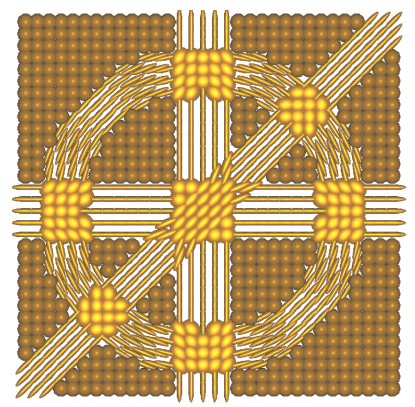

(a) Crossing fiber phantom (from 14 visualized using [4]).
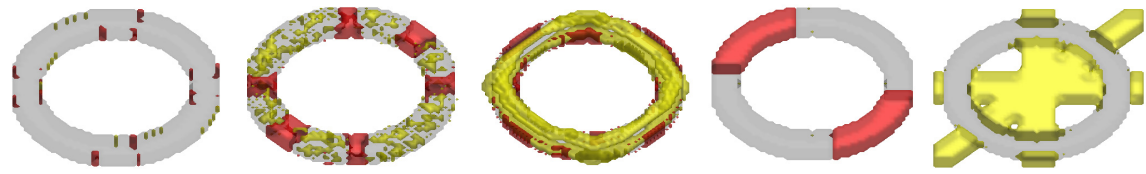

Proposed

Niethammer et al. 15

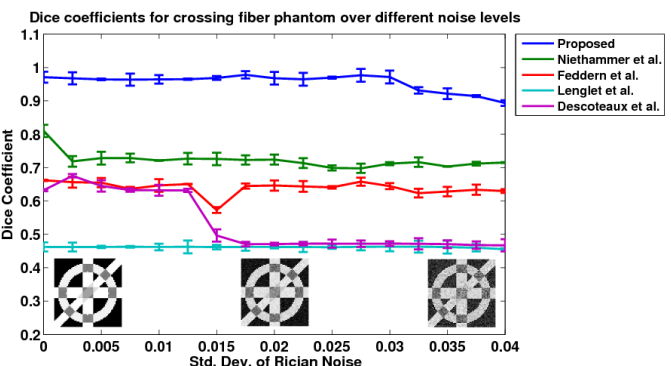

(b) DSC for phantom at different noise levels. Inset are FA maps for (left to right) images with $\sigma=0,0.02$, and 0.04 .

(c) Sample segmentation results for all methods at noise level $\sigma=0.01$. Oversegmentation is shown in yellow while under-segmentation is shown in red. The ground truth segmentation is shown in gray.

Fig. 2. Segmentation results for the ring tract in (a). Note that we obtain significantly higher Dice coefficients than competing methods as we are able to better model curved tracts and fiber crossings. Further, our approach generates consistent results across various noise levels.

\section{$3 \quad$ Experimental Setup and Results}

To evaluate the effectiveness of our segmentation approach, we perform two quantitative experiments, one on the synthetic phantom presented in 14 and another on 18 cingulum bundles from dMRI scans from the IXI database 1 . In both cases, resulting segmentations were compared to expert-drawn manual segmentations using the Dice similarity coefficient (DSC). The segmentation algorithms from [9 11, 15] are used as comparison methods. In all cases, $k=5$, $\epsilon=\exp (-4)$ and the $\log$-Euclidean distance metric was used to compute the dissimilarity maps [1]. To ensure fairness of comparison between segmentation algorithms, we optimize the weights of all energy terms in all segmentation algorithms (e.g., $\alpha, \beta)$ using genetic algorithms. Results are shown for the weights that produce the maximum DSC.

Phantom Experiment: Figure 2(a) displays the middle slice of the synthetic phantom from [14]. We seek to segment the ring tract in the phantom in order to test our algorithm's ability to handle both tract curvature and crossing regions. We further test the impact of image noise by adding Rician noise of different

\footnotetext{
${ }^{1}$ http://brain-development.org
} 


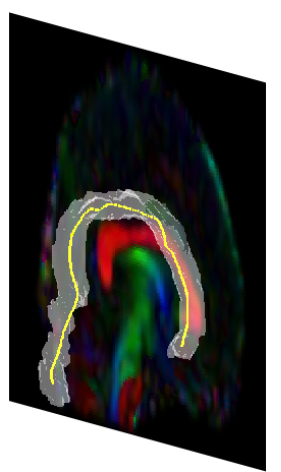

(a) Sample anchor curve (yellow) and ground truth segmentation (gray) of a cingulum bundle.

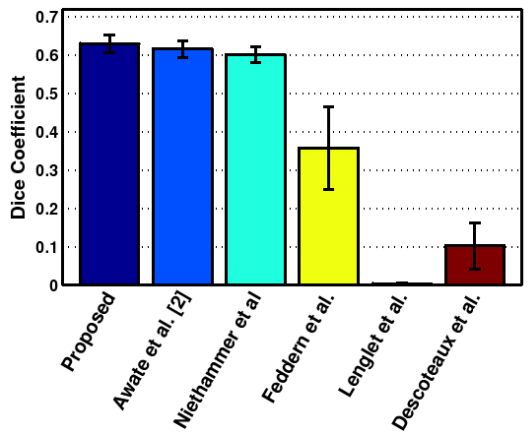

(b) Dice coefficients for all algorithms over 18 cingulum bundles. Results for Awate et al. are over only 2 datasets (taken from [2]). Although Neithammer et al.'s DSC is comparable, it suffered from localized under-segmentation in difficult areas (see text and Fig. 3(c)).

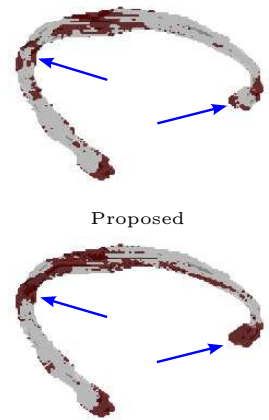

Niethammer et al. 15

(c) Sample cingulums (gray) with under-segmentation shown in red.

Fig. 3. Results on the segmentation of cingulum bundles from real dMRI scans. A sample is shown in (a). Note that we obtain significantly higher Dice coefficients than competing methods (largest $p=0.0298$ ). For the methods that were able to segment the cingulum, we were better able to reduce under-segmentation as highlighted by the blue arrows in (c).

magnitudes to the phantom. Twenty-five noisy images are generated for each noise level and all competing segmentation methods are applied.

Figure 2(b) shows the resulting DSC for each algorithm and noise level. Note that our approach significantly outperforms those algorithms presented in 9 -11, 15]. The reasons for this improvement can be seen in Figure 2(c). The approach of Niethammer et al. [15], which assumes a piecewise constant image after rotating tensors to the anchor curve's Frenet frame, has difficulty segmenting the crossing regions where the global piecewise constant assumption does not hold. However, our assumption of cross-sectional piecewise constancy still holds in these regions, resulting in a more accurate segmentation. Further, Niethammer et al. rely only on the primary eigenvector for segmentation, leading to over-segmentation leaking into isotropic regions in which the primary eigenvector may align with those within the segment of interest. Meanwhile, the geodesic active contours approach of Feddern et al. [10] is limited by poor edge information due to image noise. Finally, the piecewise constant segmentation approaches of Descoteaux et al. [9] and Lenglet et al. 11] (the latter of which includes a geodesic active contour edge term to the segmentation energy) fail to model the tensor image appropriately, leading to poor segmentations. Our approach avoids these problems by applying the piecewise constant image model on a per cross-section basis.

Real Data Experiment: We employ 18 expertly-drawn manual segmentations of cingulum bundles from 9 dMRI scans as ground truth segmentations to test the accuracy of our algorithm on real data. Figure 3(a) shows a representative 
example of the region of interest and its corresponding anchor curve. Note that the curvature of the cingulum makes a piecewise constant function a poor choice for modelling diffusion across the whole bundle.

Figure 3(b) shows the resulting DSC for all 18 cingulum segmentations. Note that our proposed approach performs better than the competing methods, even beating the average 0.615 DSC reported on comparable data for only two cingulum bundles in [2]. The piecewise constant approaches in [9, 11] failed to segment the cingulum. Instead, the segmentation leaked and delineated the corpus callosum seen in red in Figure 3(a), Meanwhile, the geodesic active contours approach of Feddern et al. [10] showed difficulty dealing with noisy edge information, leading to over-segmentation. The closest competing method to ours is that of Niethammer et al. [15], one sample of which is shown in Figure 3(c). Although the DSC values are somewhat comparable (Fig. 3(b)], the increased accuracy of our method was significant $(p=0.0298)$. Further, our approach showed consistent (i.e., over all 18 tracts) reduction in the amount of under-segmentation compared to [15]. This reduction, likely due to our use of a more localized image appearance model, was most pronounced around the genu and splenium (as highlighted by the blue arrows). Quantitatively, we observed a significant reduction of $10.48 \%$ in the number of under-segmented voxels (Niethammer et al. [15]: $\mu=1306.8$ voxels, $\sigma=362.8$ voxels; Proposed: $\mu=1169.8$ voxels, $\sigma=381.9$ voxels. $p=0.018$ ).

\section{Conclusion}

We proposed herein a cross-sectional piecewise constant model for diffusion MRI segmentation, allowing us to combine local diffusion information with global shape information. Using an "anchor curve" obtained via tractography, we are able to generate cross-sections of the tract and apply the piecewise constant model at that local level. We have shown that the resulting segmentation algorithm is better capable of handling curved tracts and crossing regions than many competing methods [2, 9 11, 15]. Future work will focus on determining whether these results remain consistent if we change the diffusion model (e.g., from tensor to ODF).

Acknowledgements. BGB was supported by both IODE Canada and the Government of Alberta. GH was parially supported by NSERC. This research has been enabled by the use of computing resources provided by WestGrid and Compute/Calcul Canada.

\section{References}

1. Arsigny, V., Fillard, P., Pennec, X., Ayache, N.: Log-Euclidean metrics for fast and simple calculus on diffusion tensors. Mag. Res. Med. 56, 411-421 (2006)

2. Awate, S.P., Zhang, H., Gee, J.C.: A fuzzy, nonparameteric segmentation framework for DTI and MRI analysis with applications to DTI-tract extraction. IEEE Trans. Med. Imag. 26(11), 1525-1536 (2007) 
3. Barbieri, S., Bauer, M.H., Klein, J., Nimsky, C., Hahn, H.K.: A variational, nonparametric approach to the fuzzy segmentation of diffusion tensor images. In: Proceedings of MICCAI Workshop on Computational Diffusion MRI (CDMRI), pp. 134-145 (2010)

4. Barmpoutis, A., Vemuri, B.C., Shepherd, T.M., Forder, J.R.: Tensor splines for interpolation and approximation of DT-MRI with applications to segmentation of isolated rat hippocampi. IEEE Trans. Med. Imag. 26(11), 1537-1546 (2007)

5. Barmpoutis, A., Hwang, M.S., Howland, D., Forder, J.R., Vemuri, B.C.: Regularized positive-definite fourth order tensor field estimation from DW-MRI. NeuroImage 45, S153-S162 (2009)

6. Booth, B.G., Hamarneh, G.: Exact integration of diffusion orientation distribution functions for graph-based diffusion MRI analysis. In: Proceedings of IEEE ISBI, pp. 935-938 (2011)

7. Bresson, X., Esedoglu, S., Vandergheynst, P., Thiran, J., Osher, S.: Fast global minimization of the active contour/snake model. J. Math. Imag. Vis. 28(2), 151-167 (2007)

8. Chiang, M.-C., Klunder, A.D., McMahon, K., de Zubicaray, G.I., Wright, M.J., Toga, A.W., Thompson, P.M.: Information-theoretic analysis of brain white matter fiber orientation distribution functions. In: Karssemeijer, N., Lelieveldt, B. (eds.) IPMI 2007. LNCS, vol. 4584, pp. 172-182. Springer, Heidelberg (2007)

9. Descoteaux, M., Deriche, R.: High angular resolution diffusion MRI segmentation using region-based statistical surface evolution. J. Math. Imag. Vis. 33(2), 239-252 (2009)

10. Feddern, C., Weickert, J., Burgeth, B.: Level-set methods for tensor-valued images. In: Proc. of IEEE Workshop on Geometric and Level Set Methods in Computer Vision, pp. 65-72 (2003)

11. Lenglet, C., Rousson, M., Deriche, R.: DTI segmentation by statistical surface evolution. IEEE Trans. Med. Imag. 25(6), 685-700 (2006)

12. de Luis-Garcia, R., Alberola-Lopez, C., Westin, C.F.: Segmentation of Tensor Fields: Recent Advances and Perspectives. In: Tensors in Image Processing and Computer Vision. Springer, London (2009)

13. Melonakos, J., Niethammer, M., Mohan, V., Kubicki, M., Miller, J., Tannenbaum, A.: Locally-constrained region-based methods for DW-MRI segmentation. In: Proceedings of ICCV, pp. 1-8 (2007)

14. Nazem-Zadeh, M.R., Davoodi-Bojd, E., Soltanian-Zadeh, H.: Level set fiber bundle segmentation using spherical harmonic coefficients. Computerized Medical Imaging and Graphics 34, 192-202 (2010)

15. Niethammer, M., Zach, C., Melonakos, J., Tannenbaum, A.: Near-tubular fiber bundle segmentation for diffusion weighted imaging: Segmentation through frame reorientation. NeuroImage 45, S123-S132 (2009)

16. O'Donnell, L., Westin, C.-F.: White matter tract clustering and correspondence in populations. In: Duncan, J.S., Gerig, G. (eds.) MICCAI 2005, Part I. LNCS, vol. 3749, pp. 140-147. Springer, Heidelberg (2005)

17. Vemuri, B.C., Liu, M., Amari, S.I., Nielsen, F.: Total Bregman divergence and its applications to DTI analysis. IEEE Trans. Med. Imag. 30(2), 475-483 (2011)

18. Zalesky, A.: DT-MRI fiber tracking: A shortest paths approach. IEEE Trans. Med. Imag. 27(10), 1458-1571 (2008) 